\title{
Dissipation of Solitary Wave Due To Mangrove Forest: A Numerical Study by Using Non-Dispersive Wave Model
}

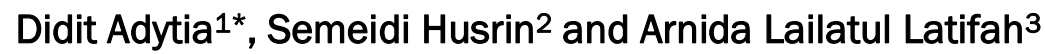 \\ ${ }^{1}$ School of Computing, Telkom University, \\ J. Telekomunikasi 1 Bandung 40257, Indonesia \\ ${ }^{2}$ Research and Development Centre for Marine and Coastal Resources Ministry of Marine Affairs and Fisheries, \\ Jl. Pasir Putih II Ancol Timur, Jakarta 14430, Indonesia \\ ${ }^{3}$ Research Center for Informatics, Indonesian Institute of Sciences, Laboratory High Performance Computing \\ J. Raya Jakarta Bogor km 47 Cibinong, Indonesia \\ Email: adytia@telkomuniversity.ac.id
}

\begin{abstract}
In this paper, we study a dissipation of solitary wave due to mangrove forest by using numerical simulation. Here, the solitary wave is chosen to represent tsunami wave form. To simulate the wave dynamic, we use the nondispersive Nonlinear Shallow Water Equations (NSWE). The model is implemented numerically by using finite volume method in a momentum conservative staggered grid. By using the proposed numerical scheme, the numerical code is able to simulate solitary wave breaking phenomenon. Wave dissipation due to mangrove forest is modelled as bottom roughness with an approximate value of manning roughness, which is derived from the classical Morisson's formula. To test the modelled dissipation by mangrove forest, we reconstruct a physical experiment in hydrodynamic laboratory where a solitary wave propagates above a sloping bottom, which has a parameterized mangrove in the shallower part. Two cases are performed to test the performance of the numerical implementation, i.e. the non-breaking and breaking solitary waves. Results of simulation agree quite well with the measurement data. The results of simulation are also analyzed quantitatively by calculating errors as well as correlation with the measurement data. Moreover, to investigate effects of wave steepness on solitary wave, to the reduction of wave energy, we perform numerical investigation. Various solitary waves with different wave steepness are simulated to see their effects on amplitude and energy reduction due to mangrove forest.
\end{abstract}

Keywords: tsunami wave, mangrove, shallow water equations, wave dissipation, finite volume, staggered grid.

\section{Introduction}

Tsunami events can be triggered by earthquakes, underwater volcanos, submarine landslide (Harbitz et al., 2014) and meteorites impact (Hills and Goda, 1998). Impact of tsunami can be very devastating especially to the coastal communities who live in areas that prone to possible tsunami hazards. To reduce possible tsunami impact for the coastal communities, some tsunami mitigation can be implemented by local authorities such as the building of tsunami breakwater and sea wall as in Japan. Such structural measure can be effective for mitigation, but can relatively be very costly. Alternatively, coastal forest such as mangrove and coastal pines, can be considered as natural barrier to dissipate tsunami wave energy (DahdouhGuebas et al., 2005; Kathiresan and Rajendran, 2005; Wolanski, 2006). However, effectiveness of coastal forest to dissipate tsunami wave energy raises many research questions, such as effective width of the coastal forest that needed to dissipate certain tsunami wave, effects of bathymetry and topography in the coastal forest to dissipate wave energy, relation between wave steepness with respect to energy dissipation by mangrove forest, and optimal age of the mangrove forest that withstand with tsunami wave.

Physical experiments and numerical approaches have been done to model the dissipation of the wave due to coastal forest. Ismail et al. (2012) investigated the dissipation of wave due to coastal vegetation by using laboratory experiment. They found that the width of vegetation greatly influences the dissipation of wave, whereas density of the vegetation is less significant in dissipating wave. For engineering practice, investigating effects of wave dissipation due to coastal forest such as mangrove by using physical experiment, can be impractical, therefore approach via numerical simulation is preferred. Tang et al. 
(2013) uses the Nonlinear Shallow Water Equations (NSWE) to model effects of solitary wave dissipation due to coastal forest in a sloping bottom. Yao et al. (2018) study the dissipation of wave due to vegetation in a sloping beach by using extended Boussinesq. Huang et al. (2011) performed physical experiments to investigate dissipation of solitary wave due rigid vegetation in a flume with a flat bottom and followed numerical simulation by using Boussinesq type of model.

Not only the vegetation width, beach topography below the coastal vegetation also affects the incident wave directly, especially its wave steepness that eventually leads to wave breaking. Hiraishi and Harada (2003), use beaches with slope of of $0,1 / 3,1 / 50$, while Istiyanto et al. (2003) uses slope with $5^{\circ}, 10^{\circ}$, and $15^{\circ}$ or equivalent with slope of $1 / 11,1 / 5.8$, and $1 / 3.9$. In this paper, we investigate numerically relation between wave steepness of solitary wave with energy and amplitude dissipation by the presence of mangrove forest. To that end, we use the Nonlinear Shallow Water Equations (NSWE) with finite volume staggered grid scheme to represent the dynamic of wave motion. The dissipation by mangrove forest is modelled by using equivalent manning roughness that is derived by using obtained drag coefficient $C_{D}$ based on laboratory experiment by Husrin (2013). The numerical implementation of NSWE is firstly validated against available experiment of solitary wave above a sloping bottom from a hydrodynamic laboratory (Husrin et al., 2012) for both nonbreaking and breaking solitary wave. The validated numerical implementation is then used for simulating solitary waves with various wave steepness. The simulations are aimed to investigate the effectiveness of mangrove forest for dissipating wave energy in term of the width of mangrove forest.

The content of this paper is as follows. In the next section, we describe the Material and Methods that are used in this paper, i.e. firstly the Nonlinear Shallow Water Equations (NSWE) and its numerical implementation by using finite volume with momentum conservative staggered grid and the physical experiment of solitary wave dissipation by parameterized mangrove forest by Husrin et al. (2012), for validating our numerical simulation It is then followed by section of Results and Discussion where we validate the wave model numerical implementation with experimental data, and then, we modify the amplitude of the solitary wave to obtain various wave steepness, then performing wave simulations to see the effects of amplitude and energy reduction. Finally, we conclude the paper in the last section.

\section{Material and Methods}

\section{Nonlinear Shallow Water Equations}

The Nonlinear Shallow Water Equations (NSWE) is the most preferable wave model for representing propagation of long wave such as tsunami since it has a relatively simple form and is fully nonlinear. In this paper, we restrict the problem into 1 horizontal dimension (1HD) problem. Let $x$ and $t$ denote the horizontal coordinate and the time, respectively. We recall the NSWE in conservative form as follows

$$
\begin{aligned}
& \partial_{t} h+\partial_{x}(h u)=0 \\
& \partial_{t}(h u)+\partial_{x}\left(h u^{2}+\frac{1}{2} g h^{2}\right)+g h \partial_{x} d-C_{f} u|u|=0
\end{aligned}
$$

Here $h(x, t), d(x, t)$ and $u(x, t)$ denote the total fluid depth, fluid depth from still water level and the horizontal velocity, respectively, see Figure 1 . The gravity acceleration is denoted by $g=9.81 \mathrm{~m} / \mathrm{s}^{2}$. Here, the coefficient of bottom roughness is notated as $C_{f}$, that can be expressed in Manning's coefficient $n$ as $C_{f}=n^{2} g / h^{1 / 3}$. The conservative form (2.1) and (2.2) are for non-moving bottom, i.e. $\partial_{t} d=0$, which can be rewritten in a familiar form as

$$
\begin{aligned}
& \partial_{t} \eta=-\partial_{x}(h u) \\
& \partial_{t} u=-g \partial_{x} \eta-u \partial_{x} u-C_{f} u|u| / h
\end{aligned}
$$

where $\eta(x, t)$ denotes the surface elevation such that $h=\eta+d$, as illustrated in Figure 1. In this paper, the NSWE (2.3-2.4) is implemented numerically by using finite volume with momentum conservative staggered grid. The idea of the implementation is proposed by Stelling and Duinmeijer (2003), later on is used by Adytia et al. (2018), Tarwidi and Adytia (2018), where discretization grid of the horizontal velocity $u$ is placed in between discretization grid of other variables such as $\eta, h$, and $d$.

We define $\eta_{i}^{n} \approx \eta\left(x_{i}, t^{n}\right)$ and $u_{i+1 / 2}^{n} \approx$ $u\left(x_{i+1 / 2}, t^{n}\right)$ as approximations for $\eta$ and $u$ at fullgrid $x_{i}, i=1,2, \ldots N+1$, and half-grid $x_{i+1 / 2}, i=$ $1,2, \cdots, N+1$, respectively, in a domain $x \in[0, L]$, at time $t . \Delta x$ and $\Delta t$ denote the length of spatial discretization in $x$ and time $t$, respectively. A control volume in this staggered grid scheme is illustration in Figure 1, in the left part, where the cross sign denotes the half-grid, while the circle the full-grid.

Following Stelling and Duinmeije (2003), the discretized forms of the continuity eq. (2.3) is as follows

$\eta_{i}^{n+1}=\eta_{i}^{n}-\Delta t\left(\frac{{ }^{*} h_{i+1 / 2}^{n} u_{i+1 / 2}^{n}-{ }^{*} h_{i-1 / 2}^{n} u_{i-1 / 2}^{n}}{\Delta x}\right)$ 
where ${ }^{*} h_{i+1 / 2}^{n}$ should be computed by using upwind method as

$$
{ }^{*} h_{i+\frac{1}{2}}^{n}+ \begin{cases}h_{i}^{n}, & \text { if } u_{i+1 / 2}^{n} \geq 0 \\ h_{i+1}^{n}, & \text { if } u_{i-1 / 2}^{n}<0 .\end{cases}
$$

The definition (2.6) states that when the flow is propagating rightward, the value of $h$ in the left is chosen as the value of ${ }^{*} h_{i+1 / 2}^{n}$, similarly for the opposite direction. The condition guarantees nonnegative value for the total depth $h$. The momentum eq. (2.2) is computed in the half-grid similarly as (2.5) as follows:

$u_{i+1 / 2}^{n+1}=u_{i+1 / 2}^{n}-\Delta t\left[g\left(\frac{\eta_{i+1}^{n+1}-\eta_{i}^{n+1}}{\Delta x}\right)-\left(u \partial_{x} u\right)_{i+1 / 2}^{n}-\right.$ $\left.\left.\frac{c_{f}}{h} u|u|\right|_{i+1 / 2} ^{n}\right]$.

Here, the so-called advection term is calculated by using horizontal momentum $q=h u$, by using relation $u \partial_{x} u=\left[\partial_{x}(q u)-u \partial_{x} q\right] / h$, such that the term $\left(u \partial_{x} u\right)_{i+1 / 2}^{n}$ can be calculated as follows

$$
\begin{aligned}
& \left(u \partial_{x} u\right)_{i+1 / 2}^{n}=\frac{1}{\bar{h}_{i+1 / 2}^{n}}\left(\frac{\bar{q}_{i+1}^{n} \cdot{ }^{*} u_{i+1}^{n}-\bar{q}_{i}^{n} \cdot{ }^{*} u_{i}^{n}}{\Delta x}-\right. \\
& \left.u_{i+1 / 2}^{n} \frac{\bar{q}_{i+1}^{n}-\bar{q}_{i}^{n}}{\Delta x}\right)
\end{aligned}
$$

where the values of $\bar{q}_{i}^{n}, q_{i+1 / 2}^{n}$ and $\bar{h}_{i+1 / 2}^{n}$ are given by the following formula:

$$
\begin{gathered}
\bar{q}_{i}^{n}=\frac{1}{2}\left(q_{i+1 / 2}^{n}+q_{i-1 / 2}^{n}\right), \quad q_{i+1 / 2}^{n}=h_{i+\frac{1}{2}}^{n} \cdot u_{i+\frac{1}{2}}^{n} \cdot \\
\bar{h}_{i+1 / 2}^{n}=\frac{1}{2}\left(h_{i}^{n}+h_{i+1}^{n}\right),
\end{gathered}
$$

and the value of ${ }^{*} u_{i}^{n}$ is calculated analogous with ${ }^{*} h_{i+1 / 2}^{n}$ by using upwind method as

$$
{ }^{*} u_{i}^{n} \begin{cases}u_{i-1 / 2}^{n}, & \text { if } \bar{q}_{i}^{n} \geq 0 \\ u_{i+1 / 2}^{n}, & \text { otherwise }\end{cases}
$$

The bottom friction is calculated as follows:

$$
\left.\frac{C_{f}}{h} u|u|\right|_{i+1 / 2} ^{n}=C_{f} \frac{\left|u_{i+1 / 2}^{n}\right| u_{i+1 / 2}^{n+1}}{\bar{h}_{i+1 / 2}^{n}}
$$

As stated in Stelling and Duinmeijer, 2003, the Courant-Friedrichs-Lewy (CFL) condition for the system (2.5) and (2.7) is given by $0<C \leq 1$, where $C=\sqrt{g d_{0}} \Delta t / \Delta x$.

\section{Damping by vegetation}

The wave dissipation by coastal vegetation is commonly computed by using the classical formula of Morisson, 1958, in term of drag and inertia losses as given by the following formula

$$
F_{\text {tot }}=\frac{1}{2} C_{D} \rho A_{f} u^{2}+C_{M} \rho V \partial_{t} u
$$

where $F_{\text {tot }}$ is the total forces by drag and inertia forces. The first term in (2.9) is the force due to drag, with $C_{D}, \rho, A_{f}$ are drag coefficient due to coastal vegetation, water density, and area of the coastal vegetation, respectively. The other term in (2.10) is the force due to inertia, with $C_{M}$ and $V$ are the inertia coefficient and the volume of submerged body of coastal vegetation, respectively. The approximate value of $C_{D}$ and $C_{M}$ are usually derived from physical experiment from hydrodynamic laboratory. Another approach is to approximate the dissipation by coastal vegetation by using bottom roughness by mean of approximate manning number for given value of $C_{D}$. This approach is proposed by Yanagisawa et al., 2009 as follows

$$
n_{v}=\sqrt{\frac{h^{4 / 3}}{2 g V}\left(C_{D} A_{f}\right)+n_{b}}
$$

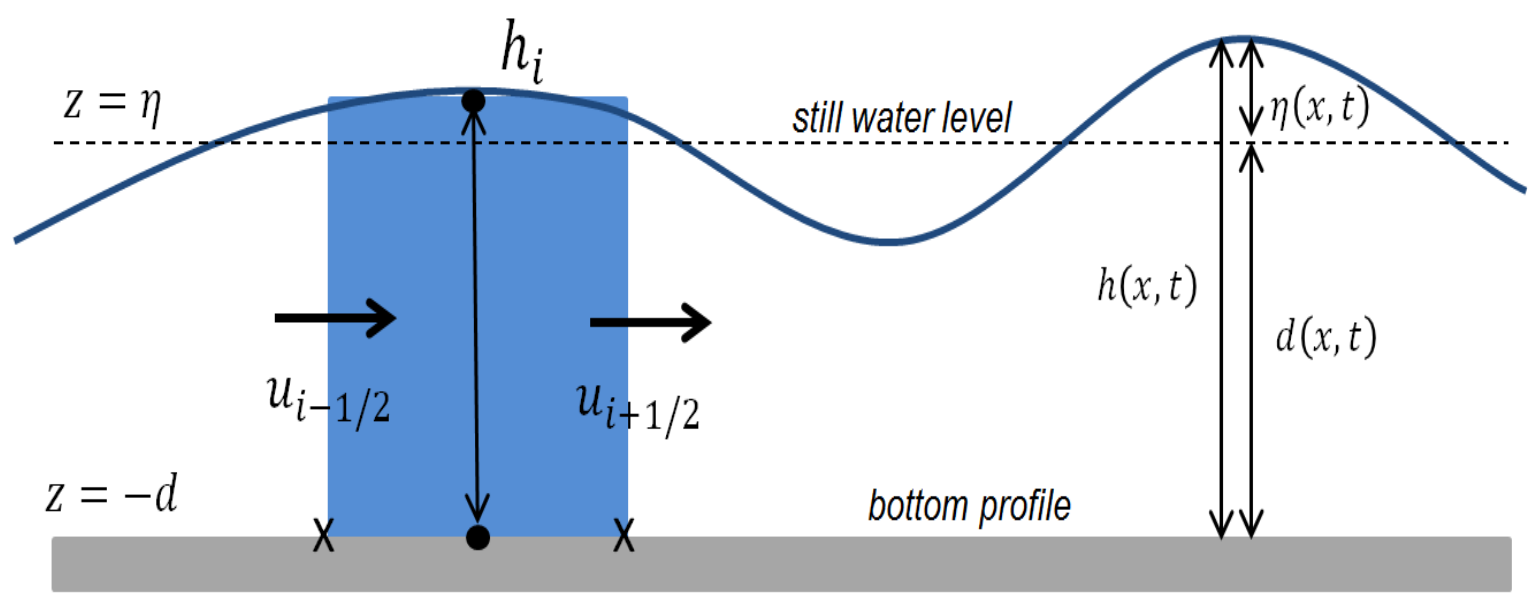

Figure 1. Illustration of variable descriptions and staggered grid scheme 
where $n_{v}$ and $n_{b}$ are manning roughness coefficient due to coastal vegetation and bottom, respectively. In this paper, the dissipation by mangrove forest is approximated by using manning roughness approach, by prescribing value of $n_{v}$ based on derived value of $C_{D}$ from physical experiment.

\section{Laboratory Experiment}

Husrin et al. (2012) perform a series of physical experiment in a hydrodynamic laboratory to investigate dissipation of many types of wave, i.e. solitary wave, regular and irregular wave, due to mangrove forest. The solitary wave is chosen for representing tsunami wave, whereas the regular and irregular waves are for storm waves. The experiments are aimed to construct standard procedure for parameterizing mangrove forest, as well as to derive drag coefficient $C_{D}$ and inertia coefficient $C_{M}$ that correspond to the parameterized mangrove forest, see Husrin (2013). In the experiment, 'real' shape of root and trunk of mangrove is parameterized as series of vertical solid cylinders as shown in Figure 2.

The experiments are performed in a twin flume basin; consisting of $1 \mathrm{~m}$ and $2 \mathrm{~m}$ wide flume, which are without and with parameterized mangrove installed, respectively, see Husrin (2013). The length of the basin in $90 \mathrm{~m}$ and is $1.2 \mathrm{~m}$ high. The facility is located in Leichtweiss - Institute (LWI), TU Braunschweig, Germany. In the twin flume, a sloping bottom with slope of $1: 20$ is installed the right part of the flume while the wave flap for wave generation is placed in the left boundary of flume, see Figure 2. The experiments here, in a scale of $1: 25$, are to represent realistic tsunami wave condition.

In total there are 50 wave gauges are installed in the twin flume to measure wave height as well as flow velocity. In this paper, we reconstruct two type of solitary wave propagation with experiment number EM1-2009070309 and EM3-
2009072202. The first experiment number is for the non-breaking case, and the other one is for the breaking case.In the experiment, solitary waves are generated by using wave flap at $x=0 \mathrm{~m}$. For the case EM1-2009070309, the wave propagating above an initial depth $d_{0}=0.565 \mathrm{~m}$ to the shallower depth $d_{1}=0.15 \mathrm{~m}$, as shown in Figure 2. Whereas for the case EM3-2009072202, $d_{0}=0.615 \mathrm{~m}$ and $d_{1}=0.2 m$ are used.

In the experiment of Husrin et al. 2012, several scenarios with various width of mangrove forest, denoted as $B$, i.e. $B=0.75,1.5$ and $3 \mathrm{~m}$. In the experiment EM1-2009070309 and EM32009072202 , the width of the mangrove forest is $B=3 \mathrm{~m}$, or in the real dimension, it represents $75 \mathrm{~m}$ long of mangrove forest. The parameterized mangrove forest is placed in $x \in[34 \mathrm{~m}, 37 \mathrm{~m}]$ as shown in Figure 3. The sloping bottom is at $x \in$ $[23.65 \mathrm{~m}, 31.98 \mathrm{~m}]$. From the experiments of Husrin (2013), drag coefficient is derived for the solitary wave, i.e. $C_{D}=1.5$. This value is then used for computing equivalent manning roughness to represent dissipation by mangrove forest by using formula (2.11), we obtained $n_{v}=0.13$. The obtained $n_{v}$ is then used for simulating effects of mangrove forest in the NSWE. Except at the mangrove forest, in the domain of computation, a uniform manning roughness is used, i.e. $n=0.013$, as suggested in Husrin (2013).

\section{Results and Discussion}

\section{Numerical simulation}

The numerical simulation is performed by using the numerical scheme that has been derived in the previous section. We consider a domain of simulation $x \in[-50 \mathrm{~m}, 60 \mathrm{~m}]$, that is discretized with $\Delta x=0.1 \mathrm{~m}$ and in time $\Delta t=0.02 \mathrm{~s}$.
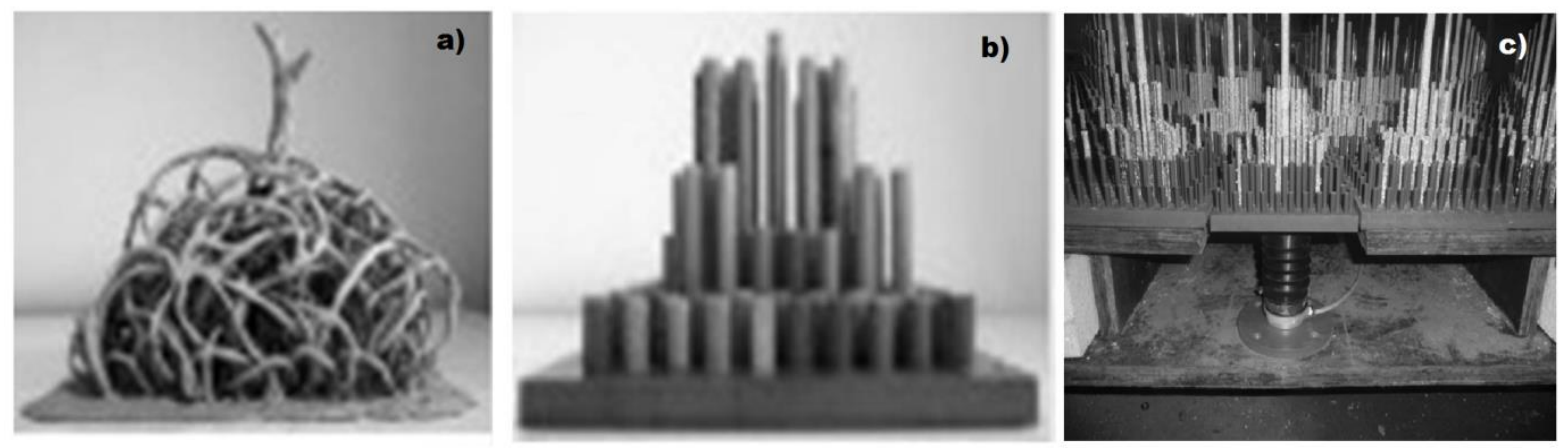

Figure 2. The 'real' shape of mangrove root and trunk (a), parameterized mangrove forest (b) and installed parameterized mangrove forest in hydrodynamic flume (c). 


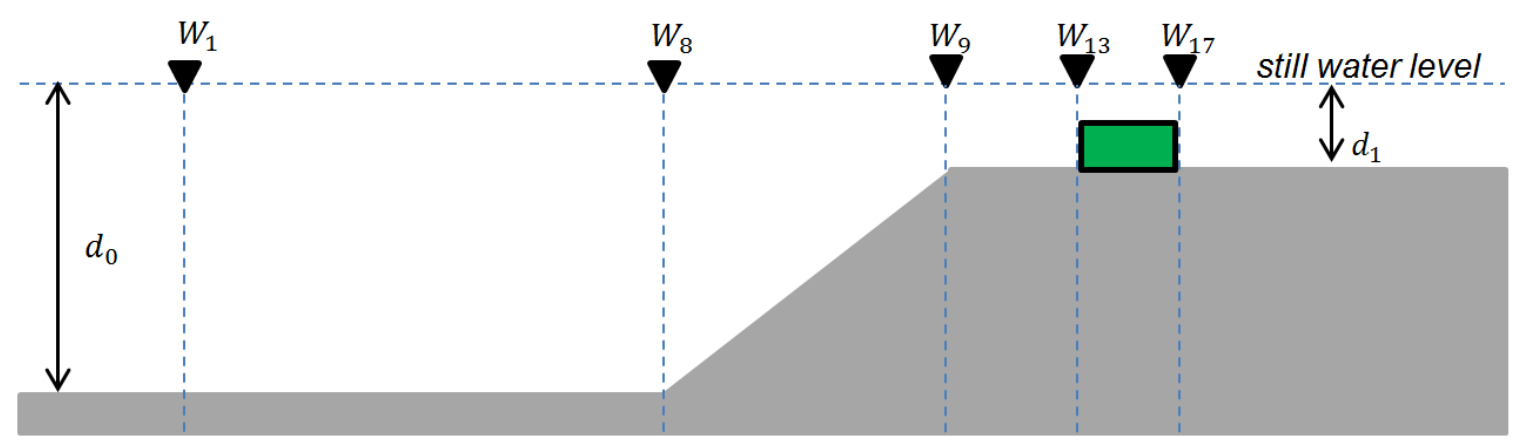

Figure 3. Layout of the physical experiment.
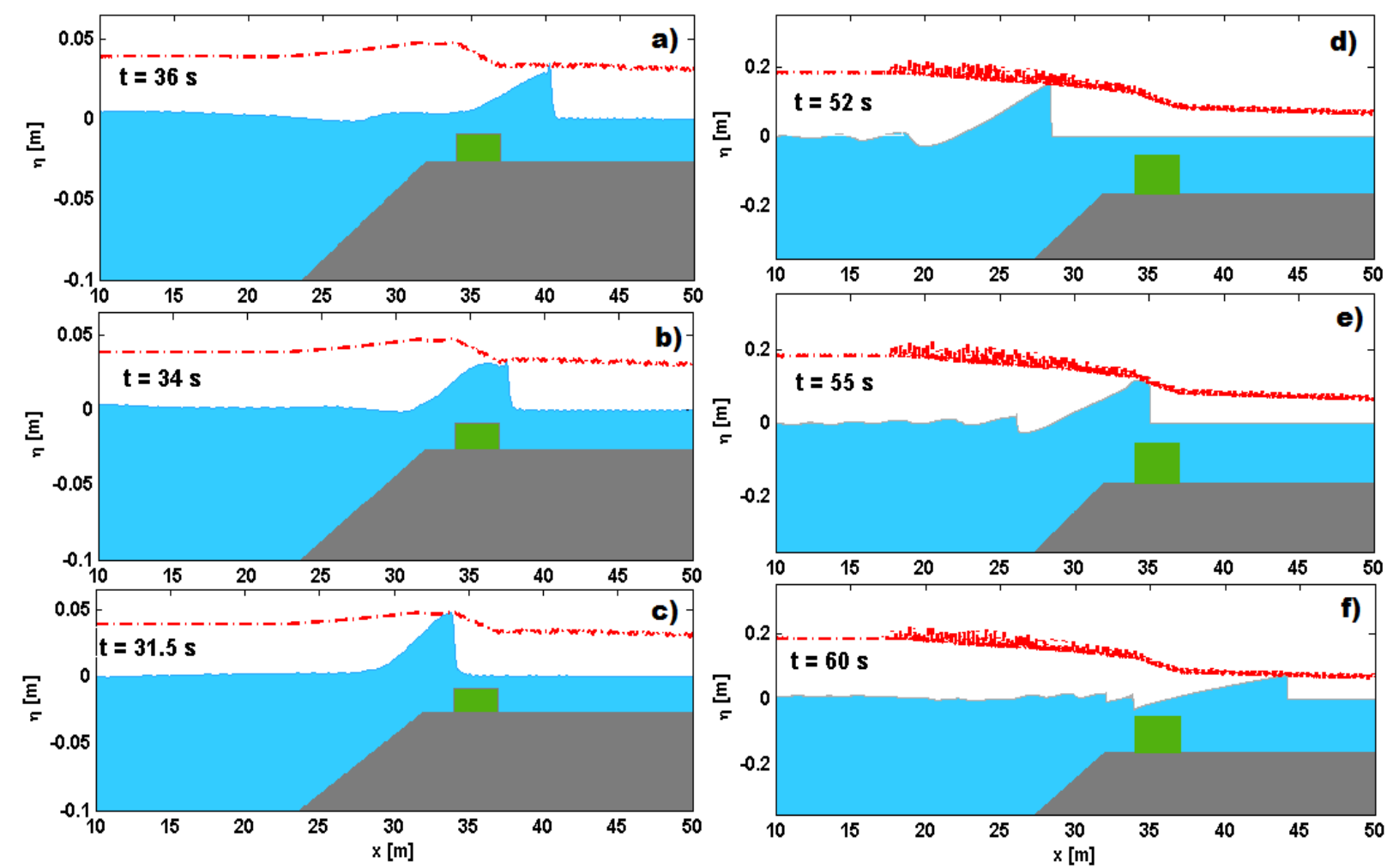

Figure 4. Snapshots of simulation for the non-breaking case (a-c) at $t=31.5,34$ and $36 \mathrm{~s}$, and for the breaking case (d-f) at $t=52,55$, and $60 \mathrm{~s}$, where red dashed lines indicate maximum wave height, while green box mangrove forest.

For generating wave to the domain of simulation, we use a signal information at wave gauge $W_{1}=10 \mathrm{~m}$ as the influx signal. We use the so-called embedded influx method (Liam et al., 2014) for influxing signal at $W_{1}$ to the domain of simulation. At both end of the domain of computation, we use a sponge layer to damp outgoing waves, with damping length of $10 \mathrm{~m}$. For the experiment EM1-2009070309 and EM32009072202, the simulations are run for $50 s$ and $90 \mathrm{~s}$, respectively.

Snapshot of the numerical simulation at $t=31.5,34$ and $36 s$ are shown in Figure 4. It is clearly seen that when the solitary wave passing mangrove area, significant amplitude reduction is detected via the plot maximum wave (indicated by dashed red lines in Figure 4). Results of simulation are compared with available experimental data at several wave gauges, i.e. $W_{4}=12.225 \mathrm{~m}, W_{5}=$ $21.415 \mathrm{~m}, W_{8}=23.650 \mathrm{~m}, W_{9}=31.98 \mathrm{~m}, W_{13}=$ $34 \mathrm{~m}$, and $W_{17}=37 \mathrm{~m}$. Signal comparisons between results of simulation with measurement data are shown in Figure 5. Qualitatively, the results of simulation show a good agreement with experimental data at all positions. 
To get a quantitative comparison between results of the simulation and the experimental data, we compare the Relative Mean Square Error (RMSE) value and Correlation Coefficient (CorrCoef) between two signals that are defined as follows:

$$
R M S E=\sqrt{\frac{\sum_{i=1}^{N}\left(y_{i}-\hat{y}_{i}\right)^{2}}{N}} \text { and } \operatorname{CorrCoef}(y, \hat{y})=
$$

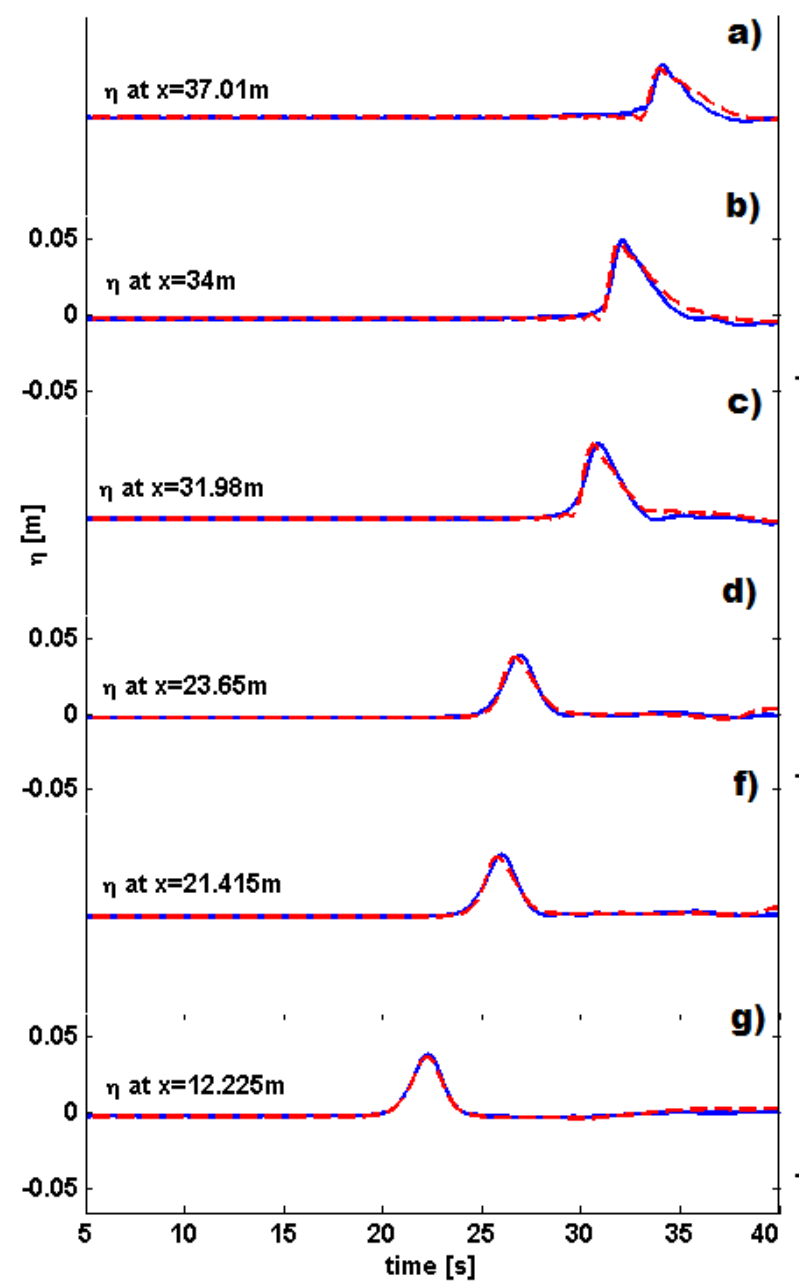

Here $y$ and $\hat{y}$ denote the experimental signal and signal from simulation, respectively. The notation |. | and $<.$, . $>$ represent $L_{2}$ norm and innerproduct, respectively. Results of the comparison are shown in Table 1. From the table, from both RMSE and correlation coefficient values show relatively very small error, i.e. RMSE $\leq 0.00194$, and Corr $\geq 0.951$ for the non-breaking case and RMSE $\leq 0.00906$, and Corr $\geq 0.798$, for the breaking case.

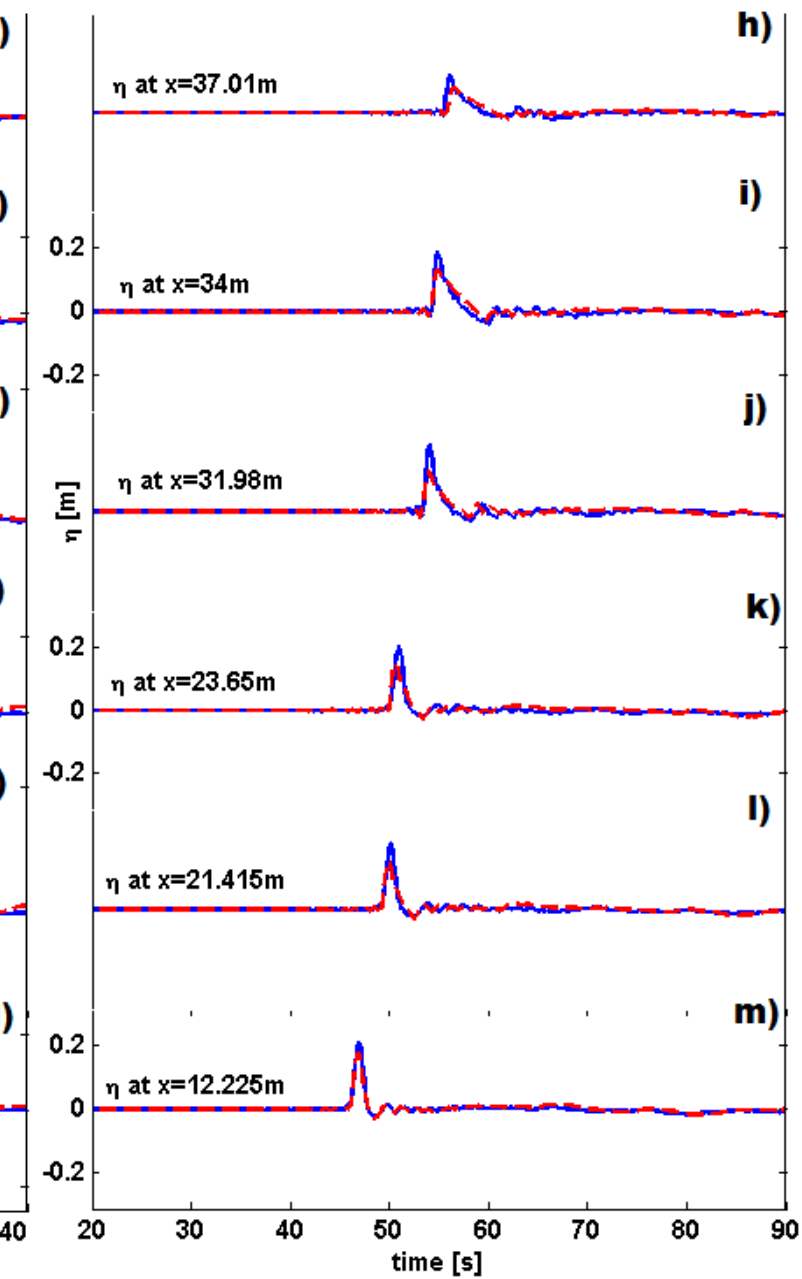

Figure 5. Comparison of signals between results of simulation (dashed red lines) and measurement (solid blue lines) at various positions for the non-breaking case ( from (a) to (g) ) and the breaking case (from (h) to (m)).

Table 1. Correlation values and RMSE and at various positions for the non-breaking and breaking case.

\begin{tabular}{cccccccc}
\hline \multicolumn{2}{c}{ Wave Gauge $(\mathrm{WG})$} & 4 & 5 & 8 & 9 & 13 & 17 \\
\hline \multicolumn{2}{c}{ Position $(\mathrm{m})$} & 12.225 & 21.415 & 23.650 & 31.980 & 34.000 & 37.000 \\
\hline \multirow{2}{*}{ RMSE } & Non-breaking & 0.00090 & 0.00109 & 0.00126 & 0.00187 & 0.00194 & 0.00185 \\
& Breaking & 0.00480 & 0.00817 & 0.00906 & 0.00857 & 0.00770 & 0.00757 \\
& Non-breaking & 0.992 & 0.988 & 0.985 & 0.977 & 0.979 & 0.951 \\
\multirow{2}{*}{ CorrCoef } & Breaking & 0.969 & 0.900 & 0.876 & 0.901 & 0.913 & 0.798 \\
\hline
\end{tabular}


The RMSE and correlation coefficient values show a good agreement between results of simulation by using staggered grid implementation of NSWE with the experimental data from hydrodynamic laboratory. Note that the NSWE is a non-dispersive wave model, but fully nonlinear model, that valid only for relatively long waves, i.e. where the ratio between wavelength $\lambda$ and water depth $d$ are larger than 20 , or $\lambda / d \geq 20$. Nevertheless, by using an appropriate numerical implementation as proposed in this paper, i.e. momentum conservative staggered grid scheme, the breaking phenomenon can be well simulated, as in the breaking case EM3-2009072202. Based on these validation results, we use the numerical model for simulating solitary waves with various wave steepness.

\section{Wave dissipation by mangrove forest}

It has been shown that the numerical implementation as described in the previous section can reconstruct the experiments of solitary wave dissipation due to the presence of mangrove forest, for both non-breaking and breaking cases. In Figure 7 , maximum surface elevation $\eta_{\max }$ for various mangrove forest length $B$, i.e. $B=0,3,6$, and $12 \mathrm{~m}$, are shown for the non-breaking case EM12009070309.

In this section, by using the same setting as the non-breaking experiment EM1-2009070309 as in the previous section, we investigate numerically length of mangrove forest that is needed for dissipating the amplitude and energy of the solitary wave. To that end, we modify the signal of experiment EM1-2009070309 at wave gauge $W_{1}$ by multiplying with amplification factors $a^{*}=0.5,1,2,3$, and 4 . The multiplication by these amplification factors leads to corresponding to wave steepness $k a=0.02,0.05,0.09,0.14$, and 0.19 , at position $W_{9}$ or before the mangrove forest, see Figure 6 . Here, $k$ and $a$ denote the wave number and amplitude, respectively, such that $k a$ indicates wave steepness, i.e. the larger the value of $k a$ the wave is steeper.

In order to investigate dissipation of amplitude and energy due to mangrove forest, for each wave steepness $k a$, we simulate the solitary waves with different mangrove forest width $B$, i.e. $B$ $=0,0.5,0.75,1.5,3,4.5,6,7.5,9,10.5,12$, and $24 \mathrm{~m}$. The mangrove is placed started from $W_{13}=$ $34 \mathrm{~m}$, as illustrated in Figure 6 . To measure the dissipation of amplitude and energy of the solitary wave, we define the amplitude and the energy reduction as follows

$$
\begin{aligned}
& A r=\frac{\left|A_{E}-A_{13}\right|}{A_{13}} \times 100 \% \\
& E r=\frac{\left|E_{E}-E_{13}\right|}{E_{13}} \times 100 \%
\end{aligned}
$$

where $A r$ and $E r$ denote the amplitude and energy reduction, respectively, $A_{E}$ and $E_{E}$ denote the amplitude and energy at the end of the mangrove forest $X_{E}, A_{13}$ and $E_{13}$ denote the amplitude and energy at wave gauge $W_{13}$ or at the beginning of the mangrove forest, respectively, illustrated Figure 6. In Figure 8, the amplitude and energy reduction, $\mathrm{Ar}$ and $E r$, are shown for various wave steepness $k a$ as function of mangrove forest width $B$ that is normalized with incoming solitary wavelength $\lambda=6.4 \mathrm{~m}$. As the energy is proportional to the quadratic of amplitude, the characteristic of $A r$ is similar with $\mathrm{Er}$. For lower $\mathrm{ka}$ value, or non-steep wave, the amplitude and energy reduction are relatively low, or require wider mangrove forest $B$. In opposite, steeper wave or wave with higher $\mathrm{ka}$ value, require relatively shorter mangrove forest $B$.

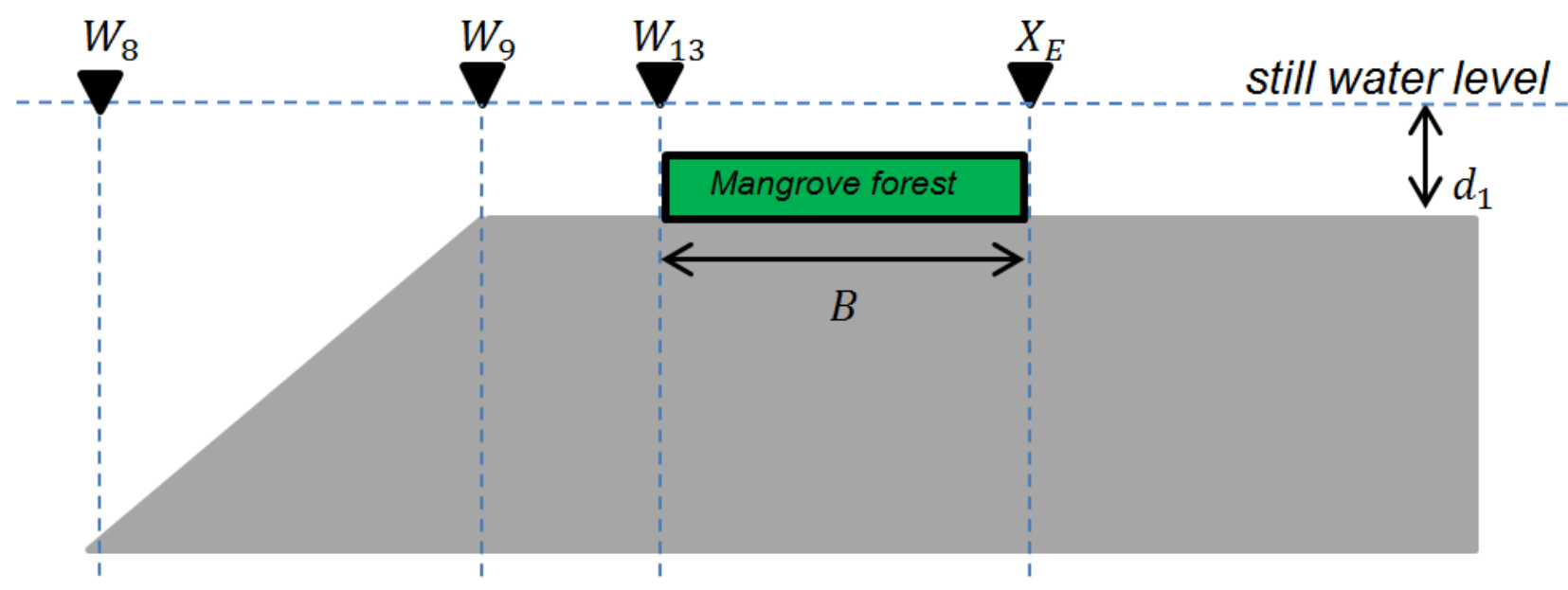

Figure. 6. Width of mangrove forest $\mathrm{B}$, started at $\mathrm{W}_{13}$. 


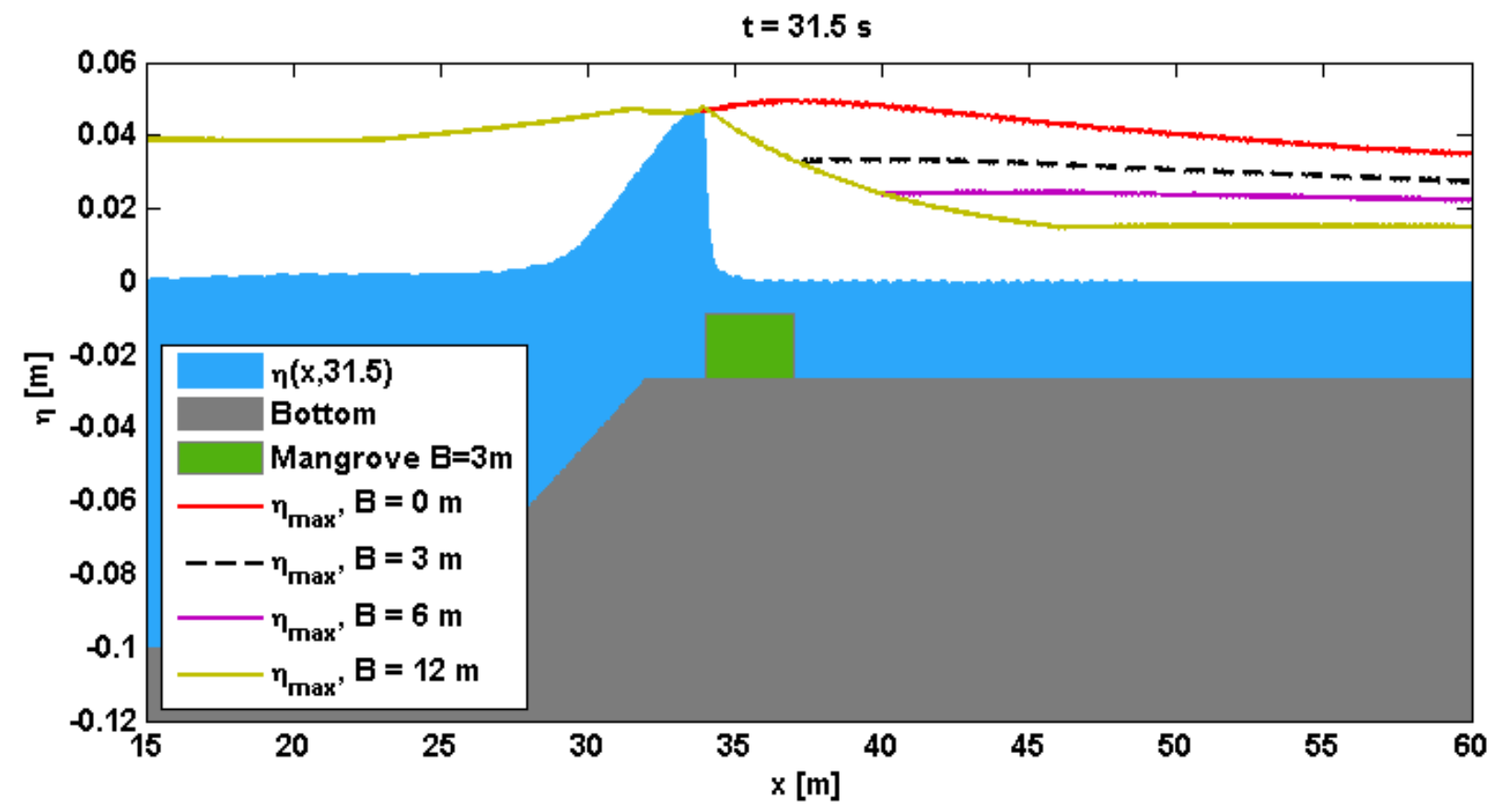

Figure. 7. Maximum surface elevation for various mangrove forest lengths B for the non-breaking case EM1-2009070309.
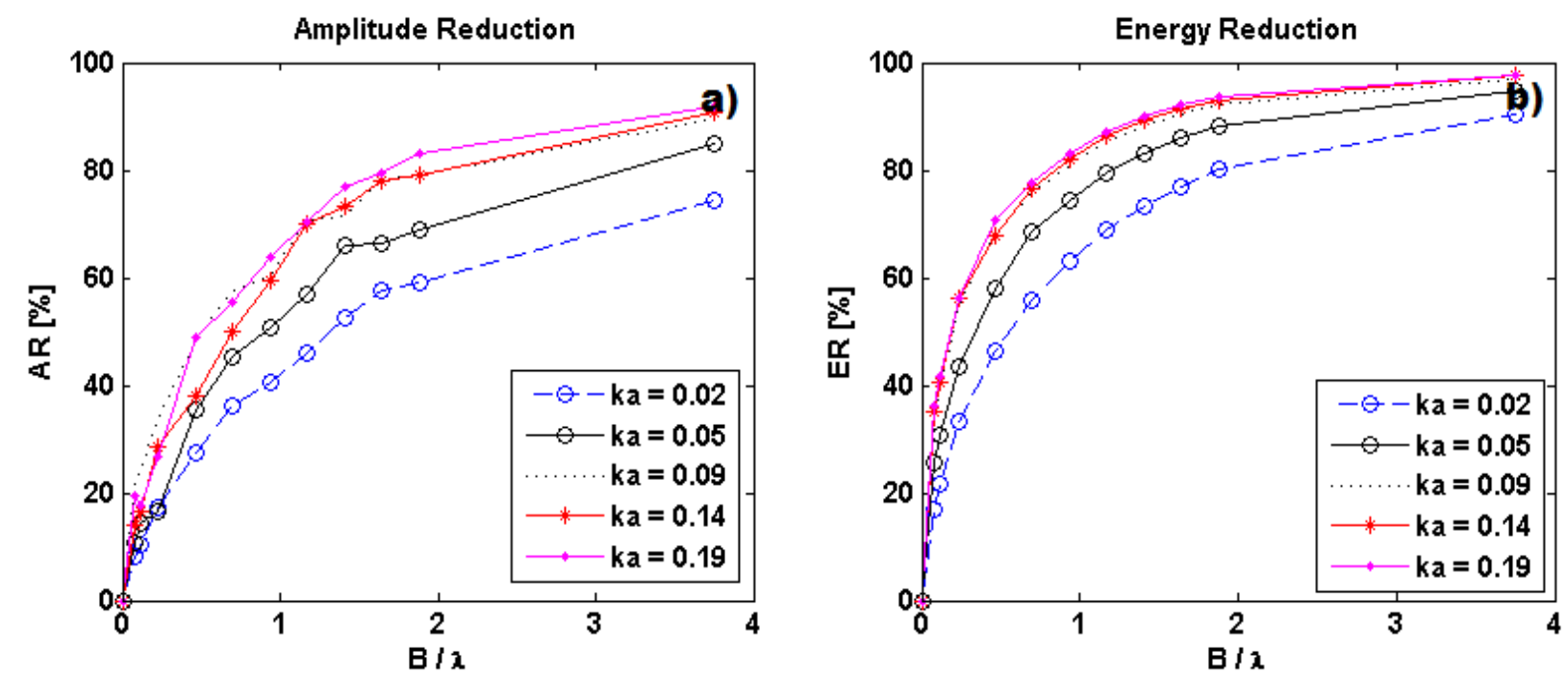

Figure. 8. Amplitude reduction (left plot) and energy reduction (right plot) as function of $\mathrm{B} / \lambda$ for various wave steepness $\mathrm{ka}$.

In this section, simulation of solitary waves with various wave steepness have been performed especially for investigating sensitivity of wave steepness of solitary waves with respect to amplitude and energy wave dissipation by mangrove forest. Based on the simulation as shown in Figure 8 , for a wave with wave steepness of $k a=0.02$, to reduce wave energy as much as $60 \%$, it requires a length of mangrove forest as long as the incoming wave length. Whereas for a steeper wave, i.e. $k a=0.19$, for a given length mangrove forest as long as the incoming wave, it may reduce the wave energy up to $80 \%$. These results conclude that the efficiency of mangrove forest for dissipating wave energy is highly depending on the incoming wave steepness, i.e. less steep wave requires longer mangrove forest to dissipate the incoming solitary wave.

\section{Conclusion}

In this paper, we have performed numerical simulations by using Nonlinear Shallow Water Equations (NSWE) with finite volume staggered grid scheme implementation for simulating dissipation of solitary wave due to mangrove forest. The presence of the mangrove is modelled as bottom roughness 
with equivalent manning roughness' coefficient, i.e. $n_{v}=0.13$, whereas in other location, it is assumed to have a uniform manning roughness $n=0.013$. The derived numerical implementation is validated against available measurement data from hydrodynamic laboratory, for both breaking and nonbreaking solitary waves. Comparison with measurement data show a good agreement between results of simulation with measured data at 6 wave gauge positions. Least good results is for the breaking case EM3-2009072202, at wave gauge $W_{17}=37 \mathrm{~m}$ or after the mangrove forest, i.e. CorrCoef $=0.79$ and RMSE $=0.00757$. This is due to the breaking process in the numerical simulation occurred earlier than the measurement data. Since the NSWE is a fully nonlinear and non-dispersive model, therefore effects of dispersion that balanced the nonlinearity effects are missing, such that the breaking process occurred earlier. An improvement is only possible when dispersive wave model is utilized. Nevertheless, for two cases of wave validation, obtained values of CorrCoef $=0.79$ and RMSE $=0.00757$ are considered relatively good fit model for engineering purpose. By using the validated model, we perform various scenarios to investigate the dependence of wave steepness with respect to the amplitude reduction and the energy reduction. To that end, we modify the amplitude of the experiment EM1-2009070309, with multiplication with amplification factor $a^{*}=$ $0.5,1,1.5,2,3$, and 4 , such that we obtain corresponding wave steepness of $k a=0.02$, $0.05,0.09,0.14$, and 0.19 . For these various wave steepness, it is concluded that less steep solitary wave requires longer mangrove forest to dissipate the amplitude as well as the energy of the wave. Whereas the steeper waves lead require less shorter mangrove forest to dissipate the amplitude and energy of the solitary wave.

\section{Acknowledgments}

The authors would like to acknowledge the Leichtweiss Institute for Hydraulic Engineering (LWI) of TU Braunschweig - Germany for the access to the experimental data of TAPFOR project.

\section{References}

Adytia, D., Tarwidi, D., Kifli, S. A. \& Pudjaprasetya, S. R.. 2018. Staggered grid implementation of 1D Boussinesq model for simulating dispersive wave J. Phys.: Conf. Ser. 971(1): 012020

Dahdouh-Guebas, F., Jayatissa, L.P., D. Di Nitto, Bosire, J.O., Lo Seen, D. \& Koedam, N. 2005. How effective were mangroves as a defence against the recent tsunami ? Current Biology 15(12):443-447.

Harbitz, C.B., Løvholt, F. \& Bungum, H. 2014. Submarine landslide tsunamis: how extreme and how likely? Natural Hazards, 72(3): 13411374.

Hills, JG. \& Goda, M P. 1998. Tsunami From Asteroid and Comet Impacts: The Vulnerability of Europe, Science of Tsunami Hazards, 16(1): 310.

Hiraishi, T. \& Harada, K., 2003. Greenbelt Tsunami Prevention in South-Pacific Region'. Report of the Port and Airport Research Institute, 42: 123.

Husrin, S., Strusinska, A. \& Oumeraci, H. 2012. Experimental study on tsunami attenuation by mangrove forest Earth Planets Space, 64(10) : 973-989.

Husrin, S. 2013. Attenuation of Solitary Waves and Wave Trains by Coastal Forests, PhD thesis: University of Braunschweig - Institute of Technology.

Huang, Z., Yao, Y., Sim, S, Y. \& Yao, Y. 2011. Interaction of solitary waves with emergent, rigid vegetation. Ocean Engineering, 38(10): 1080-1088.

Ismail, H., Abd Wahab, A.K. \& Alias, N.E. 2012. Determination of Mangrove Forest Performance in Reducing Tsunami Run-Up Using Physical Models. Natural Hazards, 63(2): 939-963.

Istiyanto, D.C., Utomo K.S. \& Suranto. 2003. Pengaruh Rumpun Bakau Terhadap Perambatan Tsunami di Pantai'(The effect of mangrove forest to the attenuation of tsunami in coastal area), Seminar Mengurangi Dampak Tsunami: Kemungkinan Penerapan Hasil Riset, BPPT - JICA, 11 March 2003, Yogyakarta

Kathiresan, K. \& Rajendran, N. 2005. Coastal mangrove forests mitigated tsunami, Est. Coast, Shelf Sci. 65(3): 601-606.

Liam, L.S., Adytia, D. \& Van Groesen, E. 2014. Embedded wave generation for dispersive surface wave models. Ocean Engineering, 80: 73-83.

Stelling, G.S., \& Duinmeijer, S.P.A. 2003. A staggered conservative scheme for every Froude number in rapidly varied shallow. Int. J. 
Numer. Methods Fluids A, 43(12): 1329-1354.

Tang, J., Causon, D., Mingham, C. \& Qian, L. 2013. Numerical study of vegetation damping effects on solitary wave run-up using the nonlinear shallow water equations, Coastal Engineering, 75: 21-28.

Tarwidi, D. \& Adytia, D. 2018. Parallelization of elliptic solver for solving 1D Boussinesq model. J. Phys.: Conf. Ser. 971(1): 012036.
Wolansky, E. 2006. Thematic paper: Synthesis of the protective functions of coastal forests and trees against natural hazards, Coastal protection in the aftermath of the Indian Ocean tsunami', FAO Regional Office for Asia and the Pacific.

Yao, Y., Tang, Z., Jiang, C., He, W. \& Liu, Z. 2018. Boussinesq Modeling of Solitary Wave Run-up Reduction by Emergent Vegetation on a Sloping Beach. J. Hydro-environment Res. 19: 78-87. 